\section{Idiosyncrasies binding assays}

Tulsi Ram Damase' \& Peter B Allen*,1

\section{ABSTRACT}

To explore thermofluorimetric analysis (TFA) in detail, we compared two related aptamers. The first, LINN2, is a DNA aptamer previously selected against EGFR recombinant protein. In this work we selected a second aptamer, KM4, against EGFRoverexpressing A549 cells. The two aptamers were derived from the same pool and bind the same target but behave differently in TFA. Our results suggest four overall conclusions about TFA of aptamers: 1 . Some aptamers show reduced fluorescence upon target binding suggesting that target-bound aptamer is not always fluorescent. 2. Many aptamers do not obey the intuitive assumptions that aptamer-target interactions stabilize a folded conformation. 3. TFA may be most appropriate for aptamers with significant double-stranded structure. 4 . Kinetic effects may be significant and the order of operations in preparing samples should be carefully optimized.

\section{METHOD SUMMARY}

We compare two aptamers generated from identical pools against EGFR to explore the advantages and limitations of thermofluorimetric analysis.

\section{KEYWORDS:}

aptamer $\cdot$ binding assay $\cdot$ cell-SELEX - EGFR • EvaGreen - hybrid fluorescence $\cdot$ melt curve analysis $\cdot$ Open $\mathrm{qPCR} \cdot \mathrm{SELEX} \cdot$ thermofluorimetric analysis

'University of Idaho, Dept of Chemistry, 875 Perimeter Dr, Moscow, ID 83844-2343, USA; *Author for correspondence: pballen@ uidaho.edu

BioTechniques 66: 00-00 (March 2019) 10.2144/btn-2018-0128

\title{
thermofluorimetric aptamer
}

GRAPHICAL ABSTRACT

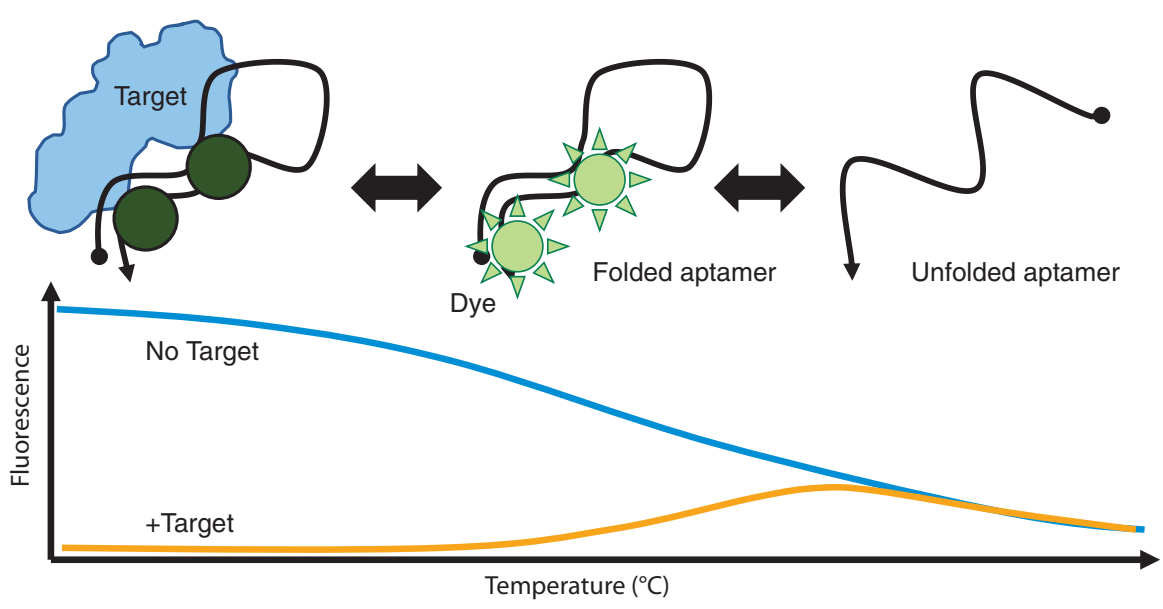

\section{INTRODUCTION}

Thermofluorimetry is an accessible technique for measuring aptamer-target equilibrium [1]. Aptamers are singlestranded DNA (ssDNA) or RNA that bind a target molecule. Aptamers have been generated using an in vitro process called SELEX (systematic evolution of ligands by exponential enrichment) since $1990[2,3]$. In SELEX, DNA that binds a target is isolated from a pool of DNA with random sequences and structures. Measuring the binding strength of the resulting aptamers is often either slow (e.g., gel shift analysis $[4,5]$ ) or is very complex and expensive (e.g., surface plasmon resonance analysis $[6,7]$, flow cytometry [8-12]). Thermofluorimetry (or melt curve analysis) can be performed with most qPCR instruments (including the inexpensive Open qPCR instrument) and can make sensitive measurements without separation of bound and unbound DNA. However, thermofluorimetry has several caveats that we explore in this work through a comparison of a published aptamer against EGFR [8] and a new aptamer from an identical parent pool.

Thermofluorimetry measures the loss of fluorescence of a dye (like EvaGreen [EG] or SYBR Green) as it dissociates from
DNA during thermal melting of doublestranded structure. It might be assumed that target-bound DNA structures should melt at a higher temperature than the unbound aptamer. However, the shape of the melt curve is dependent on the specific properties of the individual aptamer. The perturbation of this melting process by target can generate a binding isotherm. However, the interpretation of these features in terms of specific thermodynamic properties is difficult.

We have analyzed two aptamers from an identical pool to compare the thermofluorimetric properties. To select the new aptamer, we preselected the pool against recombinant EGFR for four rounds (after which the pool displayed high diversity as determined by high-throughput sequencing). We then continued the selection using A549 cells overexpressing EGFR. This is a version of 'hybrid SELEX' [13-15] and, to the best of our knowledge, represents the first cell-SELEX DNA aptamer against wild-type EGFR. EGFR is overexpressed in many cancer cells. Currently, EGFR diagnosis is based on anti-EGFR antibodies [9]. DNA is more easily synthesized with modifications like fluorophores and attachment chemistry for diagnostic applications. 
- Aptamers generated against soluble, purified, cell-surface proteins in nonphysiological conditions will often not recognize the same protein in its native conformation. This problem can be overcome by selecting aptamers for their ability to bind whole living cells under native conditions. Esposito et al. reported RNA aptamers against EGFR using cell-SELEX [16]. Tan et al. reported DNA aptamers against target human glioblastoma multiforme (GBM) cells overexpressing EGFR variant III (EGFRvIII), the most common form of EGFR mutation, using cell-SELEX [17]. Unlike the published DNA aptamer against EGFR recombinant target protein [8] the new aptamer presented here does bind to cells overexpressing EGFR.

We tested the ability of our new aptamer to bind EGFR with three methods: qPCR, flow cytometry and thermofluorimetry. Thermofluorimetry (melt curve analysis) is a relatively new method with several surprising caveats. We present data showing the importance of annealing and the order of operations as well as careful interpretation of the melting curve signal. The method of thermofluorimetry for binding assays is relatively new and has many advantages. A simple model predicts changes in the thermofluorimetric analysis (TFA) signal on target binding but is too simplistic: it ignores kinetics and the perturbations of binding by dye. We discuss the simple model and its limitations of this model in light of our data.
MATERIALS \& METHODS

Specificity test using the real-time Apta-PCR

KM4 aptamer candidate (generated by hybrid cell-SELEX; see Supplementary Figure S1) was incubated at room temperature for $2 \mathrm{~h}$ with $2 \mu \mathrm{l}$ blocked positive microspheres (EGFR-coated clear microspheres; see Supplementary Data). The incubation was followed by washing, resuspension in selection buffer and then amplification $\left(95^{\circ} \mathrm{C}, 15 \mathrm{~s} ; 64^{\circ} \mathrm{C}, 15 \mathrm{~s} ; 69^{\circ} \mathrm{C}, 30 \mathrm{~s}\right)$ on the Open qPCR. Similarly, blocked positive microspheres were incubated with scrambled DNA, 'MUT-DNA' (flanked with primer binding sites) and the same amplification analysis was performed with the Open qPCR. qPCR samples were prepared with 2x PCR Master Mix (Taq, Thermo Fisher, MA, USA) and EG dye 20x (Biotium, CA, USA).

\section{Binding assay by thermofluorimetric analysis}

Master solution containing $1 X$ EG, and $1 X$ KM4 (50 nM) was made in protein buffer (see Supplementary Data for details) and then annealed $\left(80^{\circ} \mathrm{C}, 30 \mathrm{~s}\right.$, rapidly cool to $\left.4^{\circ} \mathrm{C}\right)$. Sample containing 1X EG, 1X KM4 and EGFR was serially diluted eight times with twofold dilutions in master solution containing $1 X$ EG and 1X KM4 such that only target (EGFR) was diluted. The control experiment was carried out similarly without a target. Samples were placed in the Open qPCR and melt curve data were acquired at $1.2^{\circ} \mathrm{C}$ per min with data collection every $30 \mathrm{~s}$. We noted

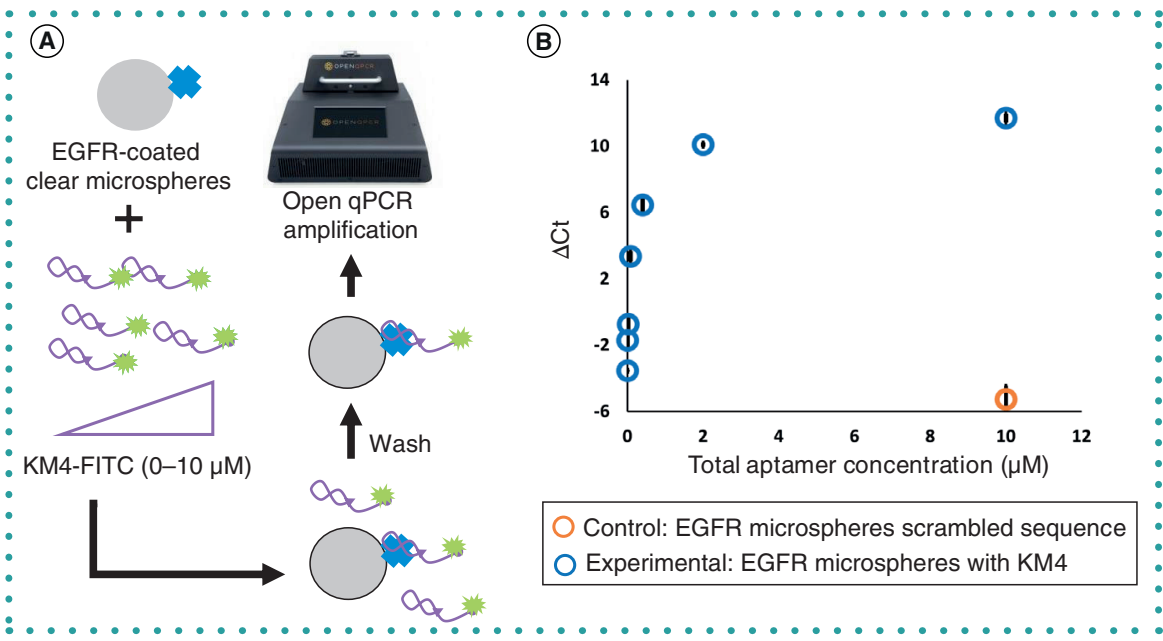

Figure 1. Real-time Apta-PCR to confirm binding of aptamer KM4 to recombinant EGFR.

(A) Schematic of the steps of Apta-PCR analysis. (B) qPCR $\triangle \mathrm{Ct}$ is plotted as a function of total aptamer concentration. $\Delta \mathrm{Ct}$ was calculated as the difference between the cycle threshold of the sample and negative control. Error bars are standard deviations of triplicates. a unique feature in the $\mathrm{dF} / \mathrm{dT}$ curve (first derivative of fluorescence with respect to temperature) in the aptamer + EGFR samples. This signal increased as a function of EGFR concentration. The importance of annealing was tested with melting curve analysis. To do so, the experiment was carried out similarly as above skipping an annealing step. A further control experiment was performed by taking nonspecific DNA 'NS-DNA' instead of aptamer KM4 in a similar way as with aptamer KM4.

\section{RESULTS \& DISCUSSION}

Screening of selected aptamers with EGFR cells

We selected a second aptamer, KM4, from the same pool to explore TFA in detail. To do so, we performed hybrid-SELEX with recombinant protein and EGFR-positive cells (Supplementary Figure S1). We evaluated the resulting aptamer candidates with qPCR (Supplementary Figure S2), fluorescence microscopy (Supplementary Figure S3), and flow cytometry (Supplementary Figure S4). We concluded that KM4 binds to EGFRpositive cells and is comparable to published aptamer LINN2 (Supplementary Figure S5). We chose the KM4 sequence for further analysis.

\section{Recombinant EGFR binding using} the qPCR

Prior to comparison of thermofluorimetric properties in detail, we needed to characterize our second aptamer, KM4. We tested binding of cell-SELEX aptamer KM4 against recombinant EGFR using real-time Apta-PCR analysis. We found that KM4 binds to recombinant EGFR-coated microspheres (Figure 1). The KM4 aptamer was synthesized with primer binding regions. We also acquired a scrambled aptamer that included primer binding regions. We incubated blocked, positive microspheres (EGFR-coated clear microspheres) with the KM4 aptamer. We varied the concentration of KM4 from 0 to $10 \mu \mathrm{M}$. We tested the negative control DNA at the high concentration of $10 \mu \mathrm{M}$. The Open qPCR computes a Ct (cycle at which fluorescence exceeds a threshold value) for each sample. We computed $\Delta \mathrm{Ct}$ by comparison to a water-only control (where water was added to the qPCR master mix instead of template). The results revealed the selected aptamer KM4 could bind to recombinant 


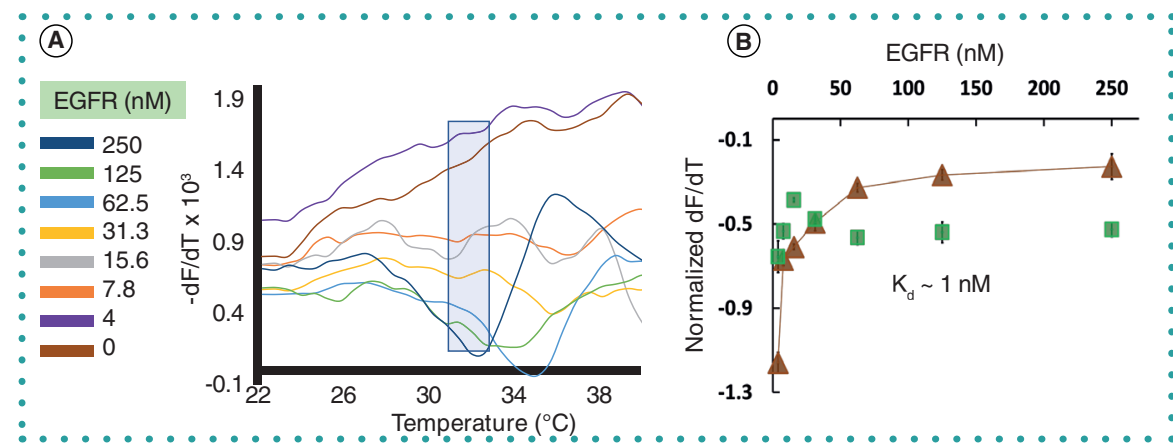

Figure 2. Thermofluorimetric analysis to determine the binding constant of aptamer KM4 to recombinant EGFR. (A) The derivative signal $(\mathrm{dF} / \mathrm{dT})$ is plotted as a function of temperature. The value of $\mathrm{dF} / \mathrm{dT}$ changes intensity as a function of EGFR concentration. (B) Comparison of binding isotherm of the aptamer (KM4) and nonspecific DNA (NS-DNA). The binding curve was generated by plotting $\mathrm{dF} / \mathrm{dT}$ versus EGFR concentration at $30-32^{\circ} \mathrm{C}$. Error bars are standard deviations of the differential signals at $30-32^{\circ} \mathrm{C}$.

- EGFR as well as EGFR-overexpressing cells.

\section{Thermofluorimetric analysis}

We used KM4 to explore the differences in TFA response compared with LINN2. Having confirmed that aptamer KM4 binds to A431 cells and recombinant EGFR, we measured the binding using thermofluorimetry (as shown in Figure 2A). These results show a melting transition that represents an analytical signal at $30-32^{\circ} \mathrm{C}$. The analytical signal corresponds to the interaction between the aptamer and target. We chose the average value of the $\mathrm{dF} / \mathrm{dT}$ signal at $30-32^{\circ} \mathrm{C}$ and plotted against EGFR concentration to create a binding curve. Depending on the aptamer, we use a temperature from $22-60^{\circ} \mathrm{C}$. Within this temperature range, we look for a distinct, changing signal as a function of target concentration. We excluded temperatures above $60^{\circ} \mathrm{C}$ (as such temperatures will likely denature aptamer and/or protein structure). In the case of the TFA shown in Figure $2,30-32^{\circ} \mathrm{C}$ was chosen by inspection to have a significant change as a function of protein concentration. The graph in Figure 2 was calculated as follows for the 250 nM EGFR case; the other concentrations were calculated similarly. A sample containing $250 \mathrm{nM}$ of EGFR concentration and $50 \mathrm{nM}$ of KM4 was subjected to thermal denaturation while monitoring fluorescence. The Open qPCR reports - dF/dT or the rate of fluorescence loss as a function of temperature. Melt curve data $(-\mathrm{dF} / \mathrm{dT})$ were collected from $30-32^{\circ} \mathrm{C}$ at five temperature points and the average and standard deviation were calculated to be $315 \pm 140$
$\mathrm{RFU} /{ }^{\circ} \mathrm{C}$. The equivalent average $-\mathrm{dF} / \mathrm{dT}$ data for the blank (0 nM EGFR plus $50 \mathrm{nM} \mathrm{KM4)}$ was calculated to be $1390 \mathrm{RFU} /{ }^{\circ} \mathrm{C}$. We normalized the $-\mathrm{dF} / \mathrm{dT}$ signal of the EGFRcontaining sample to the blank (0 nM EGFR) and calculated a ratiometric signal of $0.22 \pm$ 10 for $250 \mathrm{nM}$ EGFR and $50 \mathrm{nM}$ KM4. This ratiometric signal is plotted in terms of normalized dF/dT for clarity. Seven results are displayed because the eighth experiment (the blank) was used to normalize the other seven experiments. We fitted nonlinear regression analysis and calculated $K_{d}$ as $\sim 1 \mathrm{nM}$ as shown in Figure 2B. We performed a control experiment similarly taking NS-DNA instead of aptamer KM4 (as shown in Figure 2B, blue circles). NS-DNA does not show binding.

The measured $K_{d}$ from TFA $(\sim 1 \mathrm{nM})$ differs from that derived from cytometry $(\mathrm{Kd}=46 \mathrm{nM}$; see Supplementary Data Figure S4). There are multiple reasons to account for this difference: 1) TFA is a homogeneous, one-step technique. Flow cytometric analysis is a heterogeneous technique and requires washing. Washing might reduce the observed affinity of the aptamer. 2) In TFA, the signal-to-background ratio is aptamer-specific. Some uncertainty of the signal at low target concentrations might be the reason for the difference in $K_{d} .3$ ) Each aptamer also differs in its binding mechanism and entropically driven aptamers can show significantly higher 4) TFA uses intercalating dyes to report the interaction of aptamer and target. EG is an intercalating dye and consists of two acridine orange moieties linked with affınity at a higher temperature [18-20]. a flexible spacer. Intercalation may affect DNA structure, and bound dye may interact with the protein as well

\section{Model of TFA}

We constructed an equilibrium model to examine the thermofluorimetric behavior produced by different mechanistic assumptions. We show that an intuitive model of aptamer-target melting cannot produce the results we obtained. Under the intuitive model, a fluorescent aptamer will be stabilized by target binding. Under this model, aptamer-target complex is stabilized and will remain fluorescent at higher temperatures (compared with aptamer alone). This may be true for some aptamers but is not true for KM4 or LINN2. We suspect that the mechanism of signal generation in TFA will be specific to the aptamer sequence. To show the contrast between this predicted behavior and our observed behavior, we generated four simulated melt curves for qualitative comparison to our data.

To construct this model, we consider two simultaneous equilibria between three states: 1) target-bound, structured holoaptamer (' $\left.H^{\prime}\right)$; 2) an unbound, structured apo-aptamer ('A'); and 3) unbound, denatured, linear aptamer (' $\mathrm{L}$ '). The equilibrium between apo-aptamer and linear aptamer is assumed to be governed by a binding enthalpy and temperature-independent entropy on the order of that derived from mFold [21]. The binding energy is derived from published aptamer calorimetric data (and so is realistic but not related to this specific aptamer) [22]. The model ignores dye-DNA equilibrium and any possible kinetic effects. This equilibrium model should be considered a qualitative exploration of overall trends.

The model was defined by two equilibria: $\mathrm{K}_{\mathrm{al}}$ is the apo-linear (melting) equilibrium coefficient, and $\mathrm{K}_{\mathrm{ah}}$ is the apo-holo (binding) equilibrium coefficient:

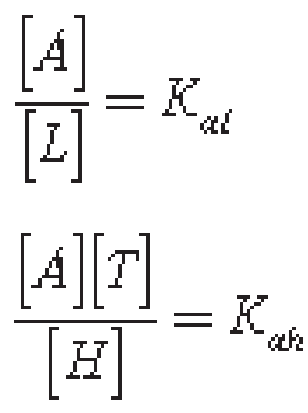


- These equilibria were constrained by the total aptamer, $A_{\text {tot' }}$ and the total target, $T_{\text {tot }}$ :

$$
[A]+[Z]+[H]=A_{\text {tot }}
$$

These four equations can be solved explicitly (see Supplementary Data, Python source code). The result is an expression for the $\mathrm{H}$ and $\mathrm{A}$ concentration as a function of $\mathrm{K}_{\mathrm{al}}, \mathrm{K}_{\mathrm{ah}}, \mathrm{A}_{\text {tot }}$ and $\mathrm{T}_{\text {tot }}$.

$$
[\Gamma]+[\mathcal{T}]=T_{\text {tot }}
$$

This equilibrium model allowed us to calculate theoretical concentrations for $\mathrm{H}$, $A$, and linear DNA at a range of target concentrations and temperatures. The result is a simulated TFA curve that can show a variety of behaviors over a reasonable range of input parameters.

We show our experimental melt curve data (Figure 3A-B) and four simulated cases for comparison (Figure $3 \mathrm{C}-\mathrm{F}$ ). We consider that the $\mathrm{H}$ may or may not be fluorescent. We also consider two scenarios for the thermodynamics of aptamer-target binding: 1) where the aptamer-target binding equilibrium coefficient is nearly constant over the relevant temperature range per known data [22]; and 2) where we contrive that the aptamer-target complex should also display melting behavior in the relevant range. The intuitive assumptions (i.e., that $\mathrm{A}$ and $\mathrm{H}$ are fluorescent; $\mathrm{K}_{\mathrm{ah}}$ is constant) produce the behavior shown in Figure 3C. In this case, aptamer binding produces increased fluorescence and an apparent increase in melting temperature. However, this simulated curve is clearly inconsistent with the data for KM4 and LINN2. Figure $3 \mathrm{~F}$ is more qualitatively consistent and is derived from the assumption that the bound aptamer is non-fluorescent and has an independent melting event. The model shows that LINN2 and KM4 do not follow intuitive assumptions about the underlying chemistry of thermofluorimetric analysis. We suggest the following considerations when analyzing TFA melt curves:

1) Some aptamers may not obey the assumption that both the $\mathrm{H}$ and $\mathrm{A}$ are highly fluorescent. We should acknowledge that either may bind intercalating dye more efficiently depending on the specific aptamer DNA sequence.

2) Some aptamers may not obey the assumption that target is released from bound aptamer at a different temperature than the denaturation of the unbound folded structure.

3) Some aptamers may produce more fluorescence than others in the presence of fluorogenic dyes. Some aptamers will therefore perform better in TFA than others. With dyes like EG, the signal-to-background ratio is better for aptamers with significant double-stranded structure (see predicted secondary structures, Supplementary Data Figure S5).

We attempted to fit the simulation to experimental TFA data to determine the thermodynamic parameters. Although the data resemble the model shown in Figure $3 F$, the fit parameters derived from that model were highly contradictory. This further indicates that the equilibrium model is not a complete description of the process. Fitting the model to the curve of the aptamer alone (0 nM EGFR) yielded a set of thermodynamic constants for the melting equilibrium. Fitting the case with $65 \mathrm{nM}$ of EGFR present yielded another set of thermodynamic constants for the melting equilibrium and constants for the binding equilibrium. These constants were irreconcilable. Fitting both simultaneously failed to converge (see Supplementary Data Figure S6).

\section{Kinetic effects on TFA}

We suspect that kinetics plays a role in the fluorescence loss during TFA. The equilibrium model ignored any kinetic effects. This may account for the quantitative differences between our model and data. Kinetic effects of dye binding, aptamer-target binding, and aptamer folding could all affect the melt curve. If the kinetics were negligible, then the order of assembly of the reaction mixture would not affect the results. However, we found that annealing the aptamer with the dye present shows a very different trend compared to annealing the aptamer alone and adding the dye. This suggests that annealing the aptamer alone may generate $a$ kinetically trapped state in which equilibrium dye binding is not obtained until the sample is heated.

We initially performed TFA by combining aptamer and EG followed by annealing. We carried out an alternate procedure by

$$
\begin{aligned}
& \left(A_{\text {tot }}^{2} \cdot K_{\alpha t}^{2}\right)+\left(2 A_{\text {tot }} \cdot K_{\alpha h} \cdot K_{\alpha t}^{2}\right)+\left(2 A_{\text {tot }} \cdot K_{\alpha h} \cdot K_{\alpha t}\right)
\end{aligned}
$$

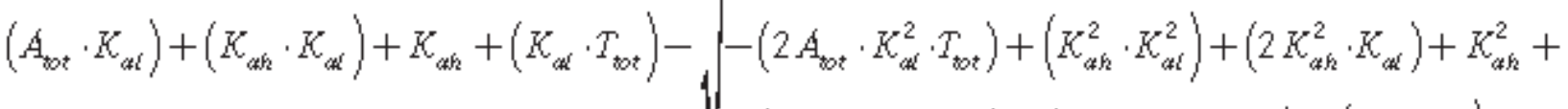

$$
\begin{aligned}
& \left(2 K_{a k} \cdot K_{a l}^{2} \cdot T_{t o t}\right)+\left(2 K_{a k} \cdot K_{a t} \cdot T_{t o t}\right)+\left(K_{a l}^{2} \cdot T_{\text {tot }}^{2}\right) \\
& {[H]=\frac{1\left(2 K_{\alpha}\right.}{2 K_{\alpha K}}}
\end{aligned}
$$

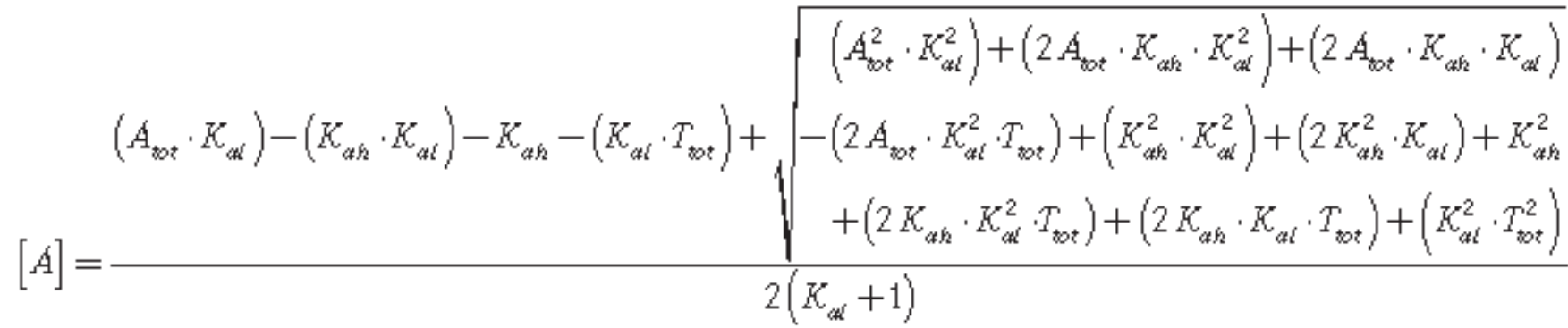




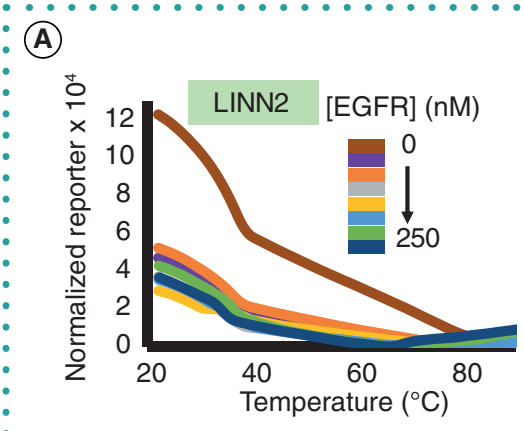

(C) Apo- and Holo-aptamer are fluorescent, aptamer binding is nearly constant

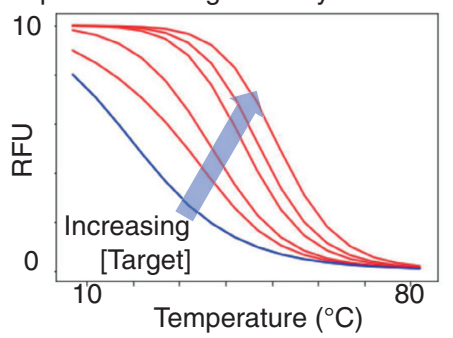

(E)

Apo- and Holo-aptamer are fluorescent, binding also shows a Tm

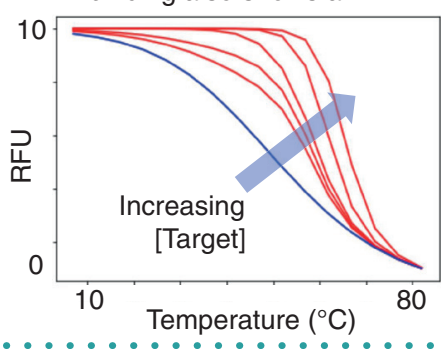

(B)

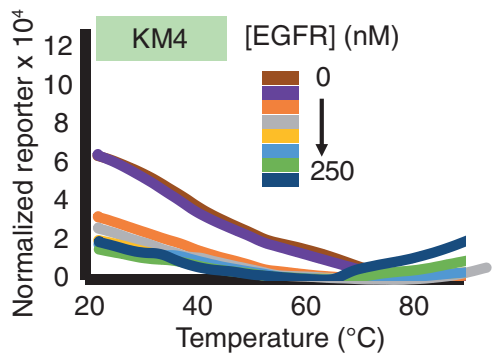

(D) Only Apo-aptamer fluorescent, aptamer binding is nearly constan

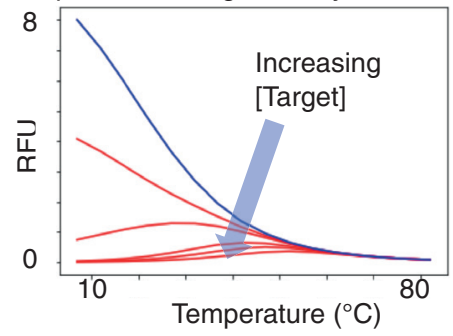

(F) Only Apo-aptamer fluorescent, binding also shows a Tm

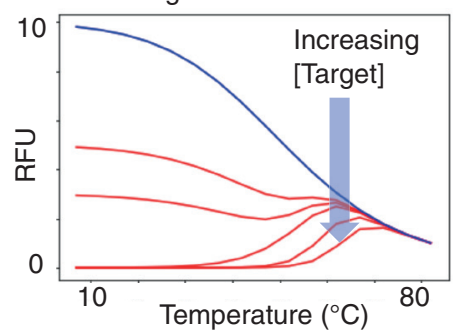

Figure 3. Qualitative comparison of experimental melt curves and simulated melt curves based on equilibrium models. (A) Thermofluorimetric analysis (TFA) of LINN2 shows fluorescence as a function of temperature. (B) TFA of KM4 shows fluorescence as a function of temperature. (C) Model when apo- and holo-aptamer are fluorescent, and target binding affinity is nearly constant. Blue line shows the aptamer only; red lines show a range of increasing target concentrations. (D) Model when only apo-aptamer is fluorescent, and target binding affinity is nearly constant. $(E)$ Model when apo- and holo-aptamer are fluorescent, and target binding also shows a Tm. (F) Model when only apo-aptamer is fluorescent, and target binding shows a Tm. Tm: Melting point.

$\checkmark$ annealing prior to adding EG. We generated a binding curve from the experimental case by plotting the average of the differential signal at $30-32^{\circ} \mathrm{C}$ against target EGFR concentration. We also generated a binding curve for the alternative case by taking of normalized average of the differential signal at $32-35^{\circ} \mathrm{C}$ against target EGFR concentration. Both were normalized with respect to blank $(0 \mathrm{nM}$ target). Annealing with EG shows a clear binding trend while the alternative did not. We interpret this to indicate that annealing with EG might set up an alternative kinetically trapped state. Such a trapped state could show a more dramatic $\mathrm{dF} / \mathrm{dT}$ signal upon rearranging at higher temperatures. It was advantageous to anneal aptamer KM4 with the dye, but every aptamer could behave differently.

\section{Implications of thermodynamic \& kinetic results}

TFA allows for the measurement of a signal from target-aptamer complexes using an intercalating dye. Our novel DNA aptamer, KM4, was selected against EGFR-overexpressing cells. This aptamer binds both cell surfaces and recombinant EGFR. The published DNA aptamer, LINN2 [8], failed to bind native cell-surface EGFR. This follows precedent set by RNA aptamers against EGFR [23].
The pair of related molecules allows for a direct comparison of aptamer idiosyncrasies when analyzed with TFA. Aptamer KM4 was not as amenable to TFA as LINN2. Its folded structure is less fluorescent in the presence of EG. Additionally, both KM4 and LINN2 show reduced EG fluorescence upon target binding. This suggests that TFA is not equally applicable to all aptamers. TFA gives a better signal-to-background ratio for aptamers having more double-stranded DNA structure.

We generated a simple equilibrium model to try to explain our TFA results. The model shows that complex melting behavior is possible. Adding kinetic effects further complicates matters. Given the complexity of the melt curves, we suggest that overinterpretation of melt curves should be avoided. Some features in the melt curve correspond to the bound complex. However, the precise thermodynamic origin of the features (e.g., stabilization of the folded structure) are probably oversimplifications.

This body of work suggests several key considerations when using TFA to perform binding assays including: mechanistic uncertainty, aptamer-dye interaction, and the possibility of kinetic effects. The common mechanistic interpretation will not be true in all cases. Only in a subset of aptamers will the folded, fluorescent aptamer be stabilized by target binding. This mechanism should not be assumed in interpreting TFA of aptamers. Aptamers will show poor performance if they have a low double-stranded structure or if their binding activity is disrupted by the intercalating dye. If mFold, NuPACK or similar software predicts few internal base pairs, then the aptamer may be a poor candidate for TFA. Finally, we note that the order of operations in assembling the reaction may affect the outcome of the experiment. This suggests that kinetic effects may be important. In addition to target concentration and buffer choice (which are common parameters for any binding assay), the order of operations in assembling samples should be carefully optimized.

Many aptamers show double-stranded structure and will be amenable to TFA, but it should not be considered universal. It may nonetheless be preferable to fluorescence anisotropy [24] or de-quenching a fluorophore [25], which require covalently v 


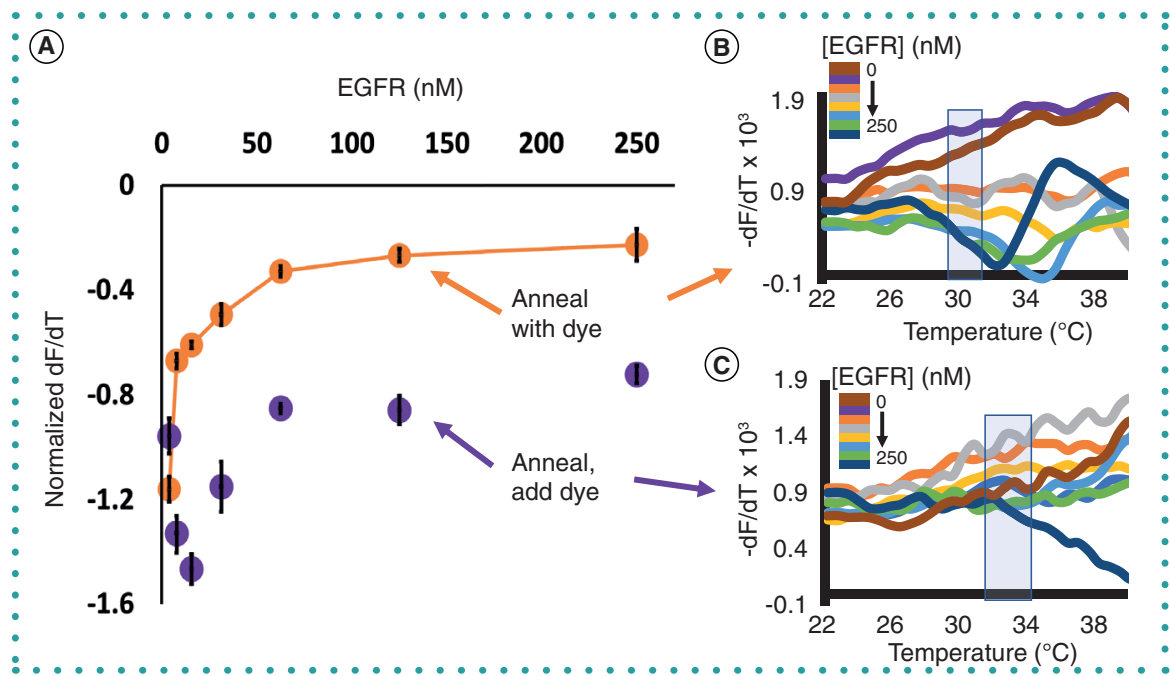

Figure 4. Kinetic effects on thermofluorimetric analysis of aptamer KM4 with recombinant EGFR (A) Binding curve generated by annealing $1 \mathrm{X}$ aptamer and EvaGreen (EG) before adding target (orange color; error bars are standard deviations of average of differential signals at $30-32^{\circ} \mathrm{C}$ ), and by annealing aptamer without EG followed by adding EG and target (violet color; error bars are standard deviations of differential signals at $\left.32-35^{\circ} \mathrm{C}\right)$. (B) The derivative signal $(\mathrm{dF} / \mathrm{dT})$ is plotted as a function of temperature for aptamer annealed with EG. (C) The derivative signal (dF/dT) is plotted as a function of temperature for aptamer annealed alone followed by EG addition.

- modified DNA and whose performance is also very specific to each aptamer. We validated the binding of aptamer KM4 to the target using a known technique, flow cytometry [8-12]. Many methods exist for measuring protein-aptamer equilibria (e.g., flow cytometry, SPR, radioactive dot blot assay) [26]. These can be used to confirm TFA results. The ubiquity and simplicity of melt curve analysis and the fact that it is a homogeneous assay all make TFA an attractive option for initial characterization of a new aptamer.

\section{AUTHOR CONTRIBUTIONS}

TRD wrote the main manuscript text, prepared figures, and conducted experiments. PBA supervised the project and helped prepare and edit the manuscript.

\section{ACKNOWLEDGMENTS}

We thank Proferssor Samir Iqbal for the kind gift of A549 cells.

\section{FINANCIAL \& COMPETING INTERESTS DISCLOSURE}

This work is supported by the National Institute of General Medical Sciences of the National Institutes of Health under Award Number P20GM104420. This published report is supported by an Institutional Development Award (IDeA) from the National Institute of General Medical Sciences of the number P30 GM103324. The content is solely the responsibility of the authors and does not necessarily represent the official views of the National Institutes of Health. The authors have no other relevant affiliations or financial involvement with any organization or entity with a financial interest in or financial conflict with the subject matter or materials discussed in the manuscript apart from those disclosed.

No writing assistance was utilized in the production of this manuscript.

\section{OPEN ACCESS}

This work is licensed under the AttributionNonCommercial-NoDerivatives 4.0 Unported License. To view a copy of this license, visit http://creativecommons.org/licenses/ by-nc-nd/4.0/

\section{SUPPLEMENTARY DATA}

To view the supplementary data that accompany this paper please visit the journal website at: www.future-science. com/doi/suppl/10.2144/btn-2018-0128

\section{REFERENCES}

1. Hu J, Kim J, Easley CJ. Quantifying aptamer - protein binding via thermofluorimetric analysis. Anal. Methods 7(17), 7358-7362 (2015).

2. Tuerk C, Gold L. Systematic evolution of ligands by exponential enrichment: RNA ligands to bacteriophage T4 DNA polymerase. Science 249(4968), 505-510 (1990).
National Institutes of Health under grant

3. Ellington AD, Szostak JW. In vitro selection of RNA molecules that bind specific ligands. Nature 346(6287), 818-822 (1990)

4. Pan Y, Duncombe TA, Kellenberger CA, Hammond MC, Herr AE. High-throughput electrophoretic mobility shift assays for quantitative analysis of molecular binding reactions. Anal. Chem. 86(20), 10357-10364 (2014).

5. Wang MS, Reed SM. Direct visualization of electrophoretic mobility shift assays using nanoparticle-aptamer conjugates. Electrophoresis 33(2), 348-351 (2012).

6. Lin $\mathrm{P}-\mathrm{H}$, Chen $\mathrm{R}-\mathrm{H}$, Lee $\mathrm{C}-\mathrm{H}$, Chang $\mathrm{Y}$, Chen $\mathrm{C}-\mathrm{S}$, Chen W$Y$. Studies of the binding mechanism between aptamers and thrombin by circular dichroism, surface plasmon resonance and isothermal titration calorimetry. Coll. Surfaces B. 88(2), 552-558 (2011).

7. Sypabekova M, Bekmurzayeva A, Wang R, Li Y, Nogues C, Kanayeva D. Selection, characterization, and application of DNA aptamers for detection of Mycobacterium tuberculosis secreted protein MPT64. Tuberculosis 104, 70-78 (2017).

8. Damase TR, Miura TA, Parent CE, Allen PB. Application of the open qPCR instrument for the in vitro selection of dna aptamers against epidermal growth factor receptor and Drosophila C virus. ACS Comb. Sci. 20(2), 45-54 (2018).

9. Wang D-L, Song Y-L, Zhu Z et al. Selection of DNA aptamers against epidermal growth factor receptor with high affinity and specificity. Biochem. Biophys. Res. Comm. 453(4), 681-685 (2014).

10. Shangguan D, Li Y, Tang $Z$ et al. Aptamers evolved from live cells as effective molecular probes for cancer study. PNAS 103(32), 11838-11843 (2006).

11. Zhang $P$, Zhao $\mathrm{N}$, Zeng $Z$ et al. Using an RNA aptamer probe for flow cytometry detection of CD30-expressing lymphoma cells. Lab. Invest. 89(12), 1423-1432 (2009).

12. Dunaway AB, Sullivan RS, Siegel KJ, Milam VT. Evaluating the dual target binding capabilities of immobilized aptamers using flow cytometry. Biointerphases 10(1), 019015 (2015).

13. Parekh P, Kamble S, Zhao N, Zeng Z, Portier BP, Zu Y. Immunotherapy of CD30-expressing lymphoma using 8909-8917 (2013).

14. Hicke BJ, Marion C, Chang YF et al. Tenascin-C aptamers are generated using tumor cells and purified protein J. Biol. Chem. 276(52), 48644-48654 (2001).

15. Sun H, Zhu X, Lu PY, Rosato RR, Tan W, Zu Y. Oligonucleotide aptamers: new tools for targeted cancer therapy. Mol. Ther. Nucleic Acids 3(8), e182 (2014).

16. Esposito CL, Passaro D, Longobardo I et al. A neutralizing RNA aptamer against EGFR causes selective apoptotic cell death. PIOS One 6(9), e24071 (2011).

17. Tan $Y$, Shi $Y$, Wu $X$ et al. DNA aptamers that target human glioblastoma multiforme cells overexpressing epidermal growth factor receptor variant III in vitro. Acta Pharmacologica Sinica 34(12), 1491-1498 (2013).

18. Amano R, Takada K, Tanaka $Y$ et al. Kinetic and thermodynamic analyses of interaction between a high-affinity RNA aptamer and Its target protein. Biochemistry 55(45), 6221-6229 (2016).

19. Sakamoto T, Ennifar E, Nakamura Y. Thermodynamic study of aptamers binding to their target proteins. Biochimie. 145, 91-97 (2018).

20. Kanakaraj I, Chen W-H, Poongavanam $\mathrm{M}$ et al. Biophysical characterization of VEGF-aHt DNA aptamer interactions. Int. J. Biol. Macromol. 57, 69-75 (2013).

21. Zuker M. Mfold web server for nucleic acid folding and hybridization prediction. Nucleic Acids Res. 31(13), 3406-3415 (2003)

22. Potty ASR, Kourentzi K, Fang $\mathrm{H}$ et al. Biophysical characterization of DNA aptamer interactions with vascular acterization of DNA aptamer interactions with vascular
endothelial growth factor. Biopolymers 91 (2), 145-156.

23. Li N, Nguyen HH, Byrom M, Ellington AD. Inhibition of cell proliferation by an anti-EGFR aptamer. PIOS One 6(6), e20299 (2011).

24. Li W, Wang K, Tan W, Ma C, Yang X. Aptamer-based analysis of angiogenin by fluorescence anisotropy. Analyst 132(2), 107-113 (2007).

25. Nutiu R, Li Y. Structure-switching signaling aptamers. $J$. Am. Chem. Soc. 125(16), 4771-4778 (2003).

26. Jing $M$, Bowser MT. A review of methods for measuring aptamer-protein equilibria. Anal Chim Acta. 686(1-2), 9-18 (2011). 